\title{
The Enhanced Care Program: Impact of a Care Transition Program on 30-Day Hospital Readmissions for Patients Discharged From an Acute Care Facility to Skilled Nursing Facilities
}

\author{
Bradley T. Rosen, MD, MBA, FACP, SFHM ${ }^{1,2 *}$, Ronald J. Halbert MD, MPH ${ }^{1,3}$, Kelley Hart, LVN', Marcio A. Diniz, PhD 1 , \\ Sharon Isonaka, MD, MS', Jeanne T. Black, PhD, MBA ${ }^{1}$
}

${ }^{1}$ Cedars-Sinai Health System, Los Angeles, California; ${ }^{2}$ David Geffen School of Medicine, University of California, Los Angeles, Los Angeles, California; ${ }^{3}$ Jonathan and Karin Fielding School of Public Health, University of California, Los Angeles, Los Angeles, California.

BACKGROUND: Increased acuity of skilled nursing facility (SNF) patients challenges the current system of care for these patients.

OBJECTIVE: Evaluate the impact on 30-day readmissions of a program designed to enhance the care of patients discharged from an acute care facility to SNFs.

DESIGN: An observational, retrospective cohort analysis of 30-day hospital readmissions for patients discharged to 8 SNFs between January 1, 2014, and June 30, 2015.

SETTING: A collaboration between a large, acute care hospital in an urban setting, an interdisciplinary clinical team, 124 community physicians, and 8 SNFs.

PATIENTS: All patients discharged from Cedars-Sinai Medical Center to 8 partner SNFs were eligible for participation.

INTERVENTION: The Enhanced Care Program (ECP) involved the following 3 interventions in addition to standard care: (1) a team of nurse practitioners participating in the care of SNF patients; (2) a pharmacistdriven medication reconciliation at the time of transfer; and (3) educational in-services for SNF nursing staff.

MEASUREMENT: Thirty-day readmission rate for ECP patients compared to patients not enrolled in ECP.

RESULTS: The average unadjusted, 30-day readmission rate for ECP patients over the 18-month study period was $17.2 \%$ compared to $23.0 \%$ among patients not enrolled in ECP ( $P$ $<$.001). After adjustment for sociodemographic and clinical characteristics, ECP patients had $29 \%$ lower odds of being readmitted within 30 days $(P<.001)$. These effects were robust to stratified analyses, analyses adjusted for clustering, and balancing of covariates using propensity weighting.

CONCLUSIONS: A coordinated, interdisciplinary team caring for SNF patients can reduce 30-day hospital readmissions. Journal of Hospital Medicine 2018;13:229235. Published online first October 4, 2017 (C) 2018 Society of Hospital Medicine ublic reporting of readmission rates on the Nursing Home Compare website is mandated to begin on October 1, 2017, with skilled nursing facilities (SNFs) set to receive a Medicare bonus or penalty beginning a year

later. ${ }^{1}$ The Centers for Medicare \& Medicaid Services (CMS) began public reporting of hospitals' 30-day readmission rates for selected conditions in 2009, and the Patient Protection and Affordable Care Act of 2010 mandated financial penalties for excess readmissions through the Hospital Readmission Reduction Program. ${ }^{2}$ In response, most hospitals have focused on patients who return home following discharge. Innovative

\footnotetext{
*Address for correspondence: Bradley T. Rosen, MD, MBA, FACP, SFHM, Cedars-Sinai Health System, 8700 Beverly Blvd. Becker B220, Los Angeles, CA 90048; Telephone: 310-423-5610; Fax: 310-423-8441; E-mail: RosenB@cshs.org Additional Supporting Information may be found in the online version of this article.
}

Received: January 9, 2017; Revised: July 10, 2017; Accepted: July 20, 2017

2018 Society of Hospital Medicine DOI 10.12788/jhm.2852 interventions have proven successful, such as the Transitional Care model developed by Naylor and Coleman's Care Transitions Intervention. ${ }^{3-5}$ Approximately $20 \%$ of Medicare beneficiaries are discharged from hospitals to SNFs, and these patients have higher readmission rates than those discharged home. CMS reported that in 2010, 23.3\% of those with an SNF stay were readmitted within 30 days, compared with $18.8 \%$ for those with other discharge dispositions. ${ }^{6}$

Some work has been undertaken in this arena. In 2012, the Center for Medicare and Medicaid Innovation (CMMI) and the Medicare-Medicaid Coordination Office jointly launched the Initiative to Reduce Avoidable Hospitalizations among Nursing Facility Residents. ${ }^{7}$ This partnership established 7 Enhanced Care and Coordination Provider organizations and was designed to improve care by reducing hospitalizations among long-stay, dual-eligible nursing facility residents at 143 nursing homes in 7 states. $^{8}$ At the time of the most recent project report, there were mixed results regarding program effects on hospitalizations and spending, with 2 states showing strongly positive patterns, 3 states with reductions that were consistent though not statistically strong, and mixed re- 
sults in the remaining states. Quality measures did not show any pattern suggesting a program effect. ${ }^{9}$ Interventions to Reduce Acute Care Transfers (INTERACT) II was a 6-month, collaborative, quality-improvement project implemented in 2009 at 30 nursing homes in 3 states. ${ }^{10}$ The project evaluation found a statistically significant, $17 \%$ decrease in self-reported hospital admissions among the 25 SNFs that completed the intervention, compared with the same 6 months in the prior year. The Cleveland Clinic recently reported favorable results implementing its Connected Care model, which relied on staff physicians and advanced practice professionals to visit patients 4 to 5 times per week and be on call 24/7 at 7 intervention SNFs. ${ }^{11}$ Through this intervention, it successfully reduced its 30-day hospital readmission rate from SNFs from $28.1 \%$ to $21.7 \%(P<.001)$, and the authors posed the question as to whether its model and results were reproducible in other healthcare systems.

Herein, we report on the results of a collaborative initiative named the Enhanced Care Program (ECP), which offers the services of clinical providers and administrative staff to assist with the care of patients at 8 partner SNFs. The 3 components of ECP (described below) were specifically designed to address commonly recognized gaps and opportunities in routine SNF care. In contrast to the Cleveland Clinic's Connected Care model (which involved hospital-employed physicians serving as the SNF attendings and excluded patients followed by their own physicians), ECP was designed to integrate into a pluralistic, community model whereby independent physicians continued to follow their own patients at the SNFs. The Connected Care analysis compared participating versus nonparticipating SNFs; both the Connected Care model and the INTERACT II evaluation relied on pre-post comparisons; the CMMI evaluation used a difference-in-differences model to compare the outcomes of the program SNFs with those of a matched comparison group of nonparticipating SNFs. The evaluation of ECP differs from these other initiatives, using a concurrent comparison group of patients discharged to the same SNFs but who were not enrolled in ECP.

\section{METHODS}

\section{Setting}

Cedars-Sinai Medical Center (CSMC) is an 850-bed, acute care facility located in an urban area of Los Angeles. Eight SNFs, ranging in size from 49 to 150 beds and located between 0.6 and 2.2 miles from CSMC, were invited to partner with the ECP. The physician community encompasses more than 2000 physicians on the medical staff, including private practitioners, nonteaching hospitalists, full-time faculty hospitalists, and faculty specialists.

\section{Study Design and Patients}

This was an observational, retrospective cohort analysis of 30-day same-hospital readmissions among 3951 patients discharged from CSMC to 8 SNFs between January 1, 2014, and June 30, 2015. A total of 2394 patients were enrolled in the $E C P$, and 1557 patients were not enrolled.

\section{ECP Enrollment Protocol}

Every patient discharged from CSMC to 1 of the 8 partner SNFs was eligible to participate in the program. To respect the autonomy of the SNF attending physicians and to facilitate a collaborative relationship, the decision to enroll a patient in the ECP rested with the SNF attending physician. The ECP team maintained a database that tracked whether each SNF attending physician (1) opted to automatically enroll all his or her patients in the ECP, (2) opted to enroll patients on a caseby-case basis (in which case an ECP nurse practitioner [NP] contacted the attending physician for each eligible patient), or (3) opted out of the ECP completely. When a new SNF attending physician was encountered, the ECP medical director called the physician to explain the ECP and offer enrollment of his or her patient(s). Ultimately, patients (or their decision-makers) retained the right to opt in or out of the ECP at any time, regardless of the decision of the attending physicians.

\section{Program Description}

Patients enrolled in the ECP experienced the standard care provided by the SNF staff and attending physicians plus a clinical care program delivered by 9 full-time NPs, 1 full-time pharmacist, 1 pharmacy technician, 1 full-time nurse educator, a program administrator, and a medical director.

The program included the following 3 major components:

1. Direct patient care and 24/7 NP availability: Program enrollment began with an on-site, bedside evaluation by an ECP NP at the SNF within 24 hours of arrival and continued with weekly NP rounding (or more frequently, if clinically indicated) on the patient. Each encounter included a review of the medical record; a dialogue with the patient's SNF attending physician to formulate treatment plans and place orders; discussions with nurses, family members, and other caregivers; and documentation in the medical record. The ECP team was on-site at the SNFs 7 days a week and on call $24 / 7$ to address questions and concerns. Patients remained enrolled in the ECP from SNF admission to discharge even if their stay extended beyond 30 days.

2. Medication reconciliation: The ECP pharmacy team completed a review of a patient's SNF medication administration record (MAR) within 72 hours of SNF admission. This process involved the pharmacy technician gathering medication lists from the SNFs and CSMC and providing this information to the pharmacist for a medication reconciliation and clinical evaluation. Discrepancies and pharmacist recommendations were communicated to the ECP NPs, and all identified issues were resolved.

3. Educational in-services: Building upon the INTERACT II model, the ECP team identified high-yield, clinically relevant topics, which the ECP nurse educator turned into monthly educational sessions for the SNF nursing staff at each of the participating SNFs. ${ }^{10}$

\section{Primary Outcome Measure}

An inpatient readmission to CSMC within 30 days of the hospital discharge date was counted as a readmission, whether the 
TABLE 1. Distribution of Patient Characteristics

\begin{tabular}{|c|c|c|c|}
\hline Patient Characteristics & $\begin{array}{c}\text { Total } \\
\mathrm{n}=3951\end{array}$ & $\begin{array}{c}\text { ECP } \\
n=2394(60.6 \%)\end{array}$ & $\begin{array}{c}\text { Comparison } \\
n=1557(39.4 \%)\end{array}$ \\
\hline Mean age at index discharge, years (SD) & $78.1(12.3)$ & $78.1(12.6)$ & $78.2(12.0)$ \\
\hline $65-84$ years & 51.4 & 50.5 & 52.9 \\
\hline$\geq 85$ years & 35.8 & 36.2 & 35.1 \\
\hline Male gender & 40.8 & 39.7 & 42.4 \\
\hline \multicolumn{4}{|l|}{ Race and/or ethnicity } \\
\hline Non-Hispanic white & 72.3 & $74.3^{\mathrm{a}}$ & $69.3^{\mathrm{a}}$ \\
\hline Black or African American & 19.1 & $18.0^{\mathrm{a}}$ & $20.8^{\mathrm{a}}$ \\
\hline Hispanic and/or Latino & 5.1 & $4.3^{b}$ & $6.3^{b}$ \\
\hline Asian & 2.9 & 3.1 & 2.8 \\
\hline Other & 0.6 & 0.4 & 0.9 \\
\hline \multicolumn{4}{|l|}{ Preferred language } \\
\hline Spanish & 3.4 & $2.8^{\mathrm{a}}$ & $4.3^{\mathrm{a}}$ \\
\hline Other & 4.2 & 3.9 & 4.6 \\
\hline \multicolumn{4}{|l|}{ Payer } \\
\hline Medicare fee for service & 45.9 & $52.9^{b}$ & $35.0^{\mathrm{b}}$ \\
\hline Dual eligible & 42.9 & $35.1^{\mathrm{b}}$ & $55.0^{\mathrm{b}}$ \\
\hline Other & 11.2 & 12.0 & 10.0 \\
\hline \multicolumn{4}{|l|}{ Hospital clinical service line } \\
\hline Orthopedic surgery & 25.7 & $28.7^{\mathrm{b}}$ & $21.1^{\mathrm{b}}$ \\
\hline General internal medicine & 20.6 & 20.1 & 21.4 \\
\hline General surgery & 8.5 & 9.1 & 7.7 \\
\hline Cardiology, medical & 8.3 & $7.4^{\mathrm{b}}$ & $9.7^{\mathrm{b}}$ \\
\hline APR-DRG severity of illness & $(n=3946)$ & $(n=2389)$ & $(n=1557)$ \\
\hline Minor & 8.1 & 8.7 & 7.1 \\
\hline Moderate & 27.1 & 26.8 & 27.7 \\
\hline Major & 43.2 & 42.9 & 43.6 \\
\hline Extreme & 21.6 & 21.6 & 21.6 \\
\hline Index discharge length of stay in days (SD) & $8.04(8.45)$ & $8.28(8.94)$ & $7.66(7.62)$ \\
\hline \multicolumn{4}{|l|}{ Index hospitalization length of stay } \\
\hline 1 to 3 days & 25.1 & 24.6 & 26.0 \\
\hline 4 to 5 days & 24.4 & 23.8 & 25.4 \\
\hline 6 to 9 days & 26.9 & 26.9 & 26.9 \\
\hline$>9$ days & 23.6 & $24.8^{\mathrm{a}}$ & $21.7^{\mathrm{a}}$ \\
\hline
\end{tabular}

aPercentages between the ECP and comparison differ at $P<.05$.

bPercentages differ at $P<.001$.

NOTE: Values are percentages unless otherwise indicated. Totals may not add to $100 \%$ due to rounding. Unless otherwise indicated, $n=3951$. Abbreviations: APR-DRG, All Patients Refined Diagnosis Related Group; ECP, Enhanced Care Program; SD, standard deviation.

patient returned directly from an SNF or was readmitted from home after an SNF discharge.

Data

ECP patients were identified using a log maintained by the ECP program manager. Non-ECP patients discharged to the same SNFs during the study period were identified from CSMC's electronic registry of SNF discharges. Covariates known to be associated with increased risk of 30-day readmission were obtained from CSMC's electronic data warehouse, including 
TABLE 2. Multivariable Logistic Regression: Odds of 30-Day Same-Hospital Readmission From SNFs

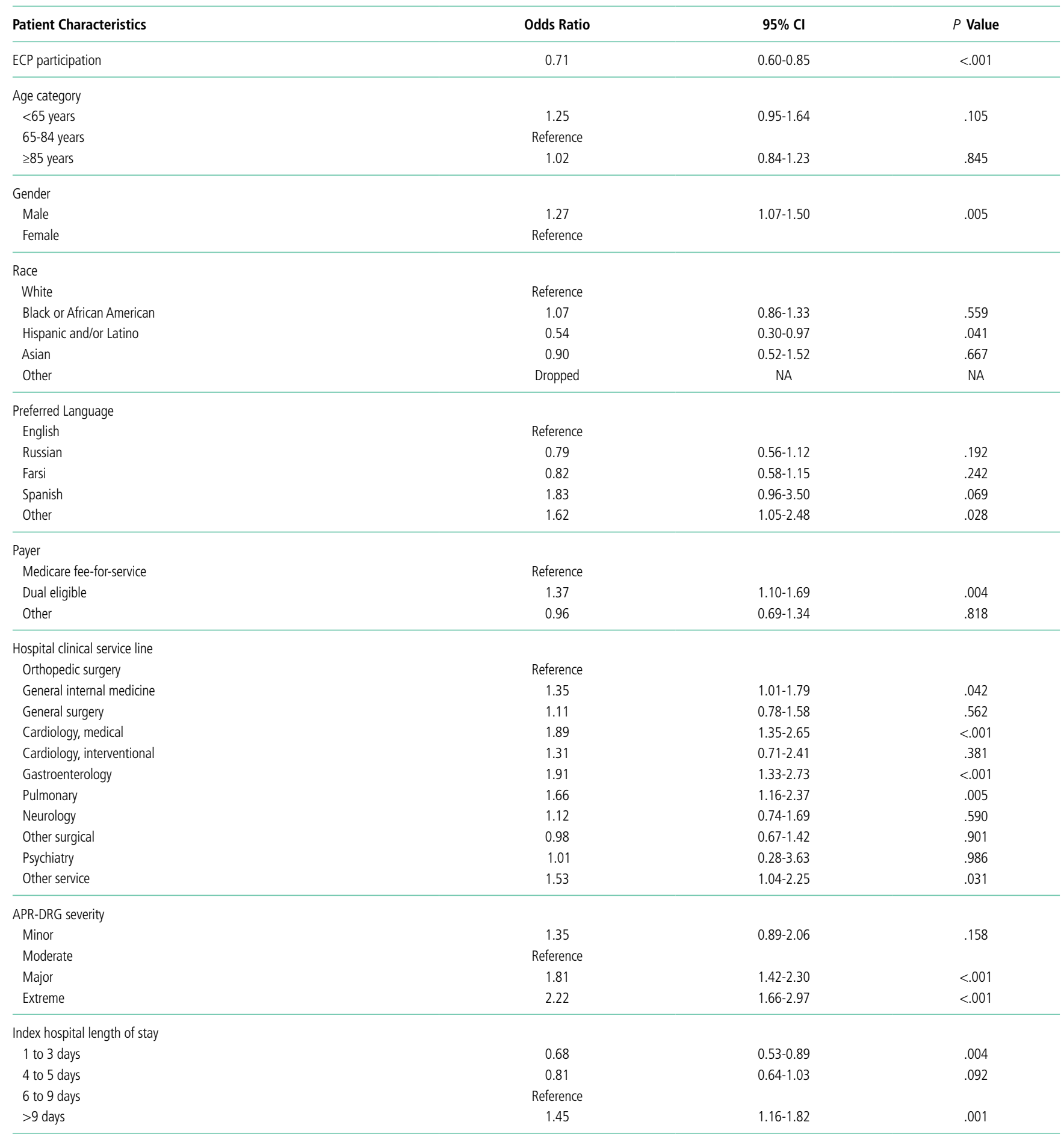

NOTE: Abbreviations: APR-DRG, All Patients Refined Diagnosis Related Group; Cl, confidence interval; ECP, Enhanced Care Program; NA, not applicable, SNF, skilled nursing facility.

demographic information, length of stay (LOS) of index hospitalization, and payer. ${ }^{12}$ Eleven clinical service lines represented patients' clinical conditions based on Medicare-Severity Diagnosis-Related groupings. The discharge severity of illness score was calculated using 3M All Patients Refined Diagnosis Related Group software, version 33. ${ }^{13}$

\section{Analysis}

Characteristics of the ECP and non-ECP patients were compared using the $\chi^{2}$ test. A multivariable logistic regression model with fixed effects for SNF was created to determine the program's impact on 30-day hospital readmission, adjusting for patient characteristics. The Pearson $\chi^{2}$ goodness-of-fit test and the 
link test for model specification were used to evaluate model specification. The sensitivity of the results to differences in patient characteristics was assessed in 2 ways. First, the ECP and non-ECP populations were stratified based on race and/or ethnicity and payer, and the multivariable regression model was run within the strata associated with the highest readmission rates. Second, a propensity analysis using inverse probability of treatment weighting (IPTW) was performed to control for group differences. Results of all comparisons were considered statistically significant when $P<.05$. Stata version 13 was used to perform the main analyses. ${ }^{14}$ The propensity analysis was conducted using $\mathrm{R}$ version 3.2.3. The CSMC Institutional Review Board (IRB) determined that this study qualified as a quality-improvement activity and did not require IRB approval or exemption.

\section{RESULTS}

The average unadjusted 30-day readmission rate for ECP patients over the 18-month study period was $17.2 \%$, compared to $23.0 \%$ for patients not enrolled in ECP $(P<.001)$ (Figure 1). After adjusting for patient characteristics, ECP patients had $29 \%$ lower odds (95\% confidence interval [Cl], 0.60-0.85) of being readmitted to the medical center within 30 days than non-ECP patients at the same SNFs. The characteristics of the ECP and comparison patient cohorts are shown in Table 1. There were significant differences in sociodemographic characteristics: The ECP group had a higher proportion of non-Hispanic white patients, while the comparison group had a higher proportion of patients who were African American or Hispanic. ECP patients were more likely to prefer speaking English, while Russian, Farsi, and Spanish were preferred more frequently in the comparison group. There were also differences in payer mix, with the ECP group including proportionately more Medicare fee-for-service (52.9\% vs $35.0 \%, P<.001)$, while the comparison group had a correspondingly larger proportion of dual-eligible (Medicare and Medicaid) patients (55.0\% vs $35.1 \%, P<.001)$.

The largest clinical service line, orthopedic surgery, had the lowest readmission rate. The highest readmission rates were found among patients with medical cardiology hospitalizations, pulmonary diseases, and gastroenterology conditions. There was a significant monotonic relationship between quartiles of index hospital LOS and 30-day readmission (Supplemental Table 1).

The largest clinical differences observed between the ECP and non-ECP groups were the proportions of patients in the clinical service lines of orthopedic surgery $(28.7 \%$ vs $21.1 \%, P$ $<.001)$, medical cardiology $(7.4 \%$ vs $9.7 \%, P<.001)$, and surgery other than general surgery (5.8\% vs $9.2 \%, P<.001)$. Despite these differences in case mix, no differences were seen between the 2 groups in discharge severity of illness or LOS of the index hospitalization. The distribution of index hospital LOS by quartile was the same, with the exception that the ECP group had a higher proportion of patients with longer LOS.

Results of the multivariable logistic regression analysis are shown in Table 2. Males had 27\% higher odds of readmission (95\% Cl, 1.07-1.50), and patients who were dually eligible for Medicare and Medi-Cal (California's Medicaid program) had

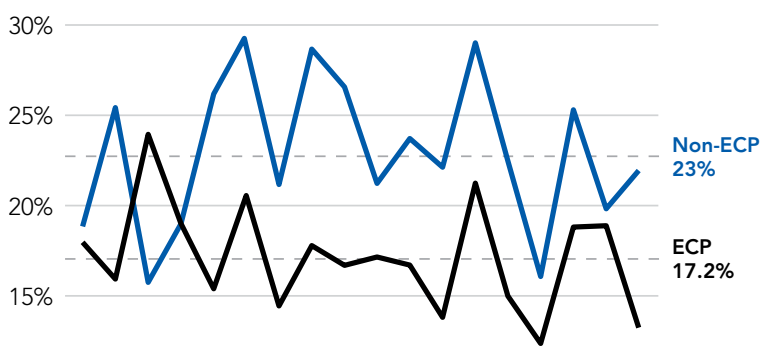

$10 \%$

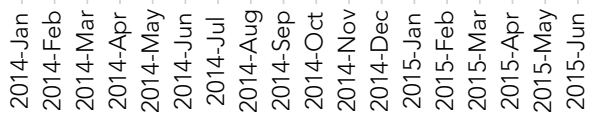

FIG 1. Monthly rate of 30-day readmissions to CSMC, ECP vs Non-ECP. Abbreviations: CSMC, Cedars-Sinai Medical Center; ECP, Enhanced Care Program; Non-ECP, Non-Enhanced Care Program

$37 \%$ higher odds of readmission (95\% Cl, 1.10-1.69). Compared with patients who had orthopedic surgery, the clinical service lines with significantly higher rates of readmission were gastroenterology (odds ratio [OR] 1.91; 95\% Cl, 1.33-2.73), medical cardiology (OR 1.89; 95\% Cl, 1.35-2.65), and pulmonary (OR $1.66 ; 95 \% \mathrm{Cl}, 1.16-2.37)$. Severity of illness at discharge and index hospital LOS were both positively associated with readmission in the adjusted analysis.

\section{Sensitivity Analyses}

The results were robust when tested within strata of the study population, including analyses limited to dual-eligible patients, African American patients, patients admitted to all except the highest volume facility, and patients admitted to any service line other than orthopedic surgery. Similar results were obtained when the study population was restricted to patients living within the medical center's primary service area and to patients living in zip codes in which the proportion of adults living in households with income below $100 \%$ of the poverty level was $15 \%$ or greater (see Supplementary Material for results).

The effect of the program on readmission was also consistent when the full logistic regression model was run with IPTW using the propensity score. The evaluation of standardized cluster differences between the ECP and non-ECP groups before and after IPTW showed that the differences were reduced to $<10 \%$ for being African American; speaking Russian or Farsi; having dual-eligible insurance coverage; having orthopedic surgery; being discharged from the clinical service lines of gastroenterology, pulmonary, other surgery, and other services; and having an index hospital LOS of 4 to 5 days or 10 or more days (results are provided in the Supplementary Material).

Figure 2 displays the 30-day readmission rate for all $\mathrm{Ce}$ dars-Sinai patients discharged to any SNF in the 3 years preceding and 4 years following the intervention. The readmission rate in the 12-month period immediately prior to the launch of the ECP was $19.6 \%$. That rate dropped significantly to $17.5 \%$ in the first 12-month period postimplementation $(P=.016)$ and to 


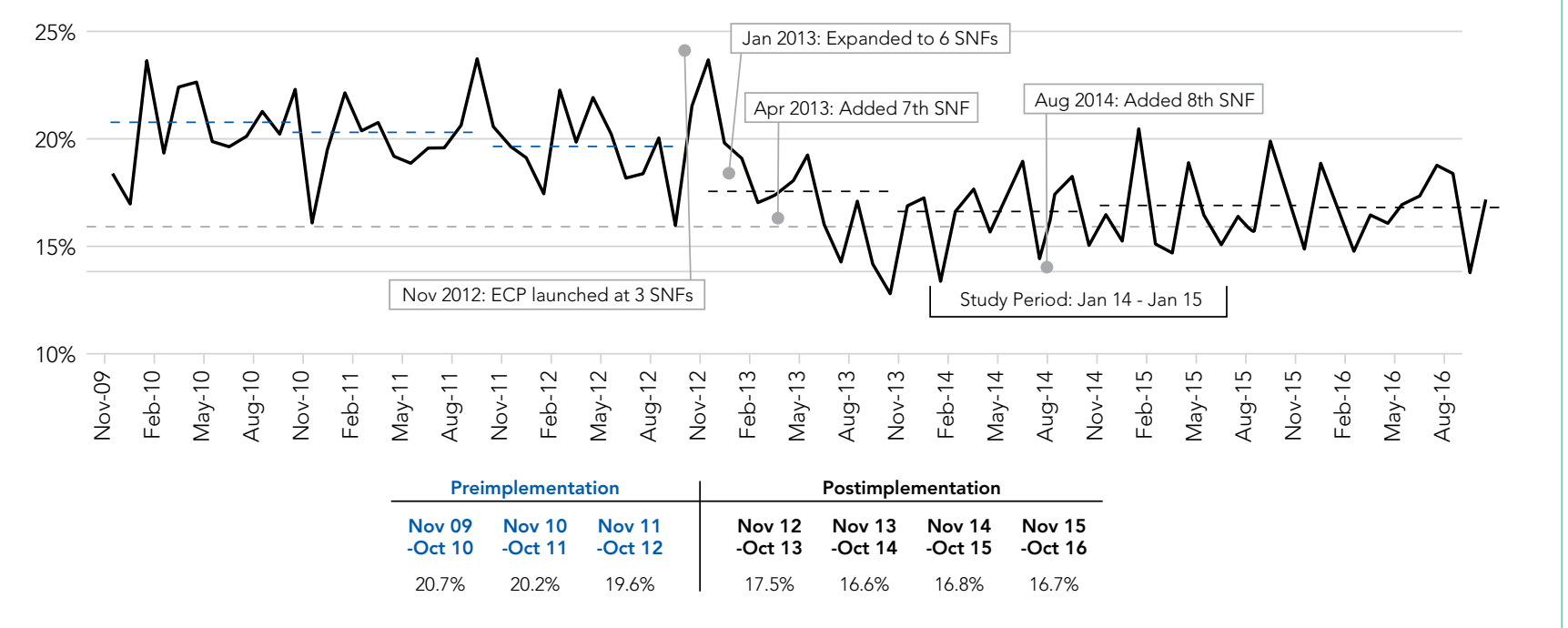

FIG 2. Mean 12-month same-hospital readmission rates of all patients discharged to SNF, pre- and postimplementation of ECP.

Abbreviations: ECP, Enhanced Care Program; SNF, skilled nursing facility.

$16.6 \%$ in the next 12 months $(P>.001$ for the overall decline). During the study period, $66 \%$ of all Cedars-Sinai patients who were discharged to a SNF were admitted to 1 of the 8 participating SNFs. More than half of those patients (representing approximately $40 \%$ of all CSMC SNF discharges) were enrolled in the ECP.

\section{DISCUSSION}

Hospitals continue to experience significant pressure to manage LOS, and SNFs and hospitals are being held accountable for readmission rates. The setting of this study is representative of many large, urban hospitals in the United States whose communities include a heterogeneous mix of hospitalists, primary care physicians who follow their patients in SNFs, and independent SNFs. ${ }^{15}$ The current regulations have not kept up with the increasing acuity and complexity of SNF patients. Specifically, Medicare guidelines allow the SNF attending physician up to 72 hours to complete a history and physical (or 7 days if he or she was the hospital attending physician for the index hospitalization) and only require monthly follow-up visits. It is the opinion of the ECP designers that these relatively lax requirements present unnecessary risk for vulnerable patients. While the INTERACT II model was focused largely on educational initiatives (with an advanced practice nurse available in a consultative role, as needed), the central tenet of ECP was similar to the Connected Care model in that the focus was on adding an extra layer of direct clinical support. Protocols that provided timely initial assessments by an NP (within 24 hours), weekly NP rounding (at a minimum), and $24 / 7$ on-call availability all contributed to helping patients stay on track. Although the ECP had patients visited less frequently than the Connected Care model, and the Cleveland Clinic started with a higher baseline 30-day readmission rate from SNFs, similar overall reductions in 30-day readmissions were observed. The key point from both initiatives is that an increase in clinical touchpoints and ease of access to clinicians generates myriad opportunities to identify and address small issues before they become clinical emergencies requiring hospital transfers and readmissions.

Correcting medication discrepancies between hospital discharge summaries and SNF admission orders through a systematic medication reconciliation using a clinical pharmacist has previously been shown to improve outcomes. ${ }^{16-18}$ The ECP pharmacy technician and ECP clinical pharmacist discovered and corrected errors on a daily basis that ranged from incidental to potentially life-threatening. If the SNF staff does not provide the patient's MAR within 48 hours of arrival, the pharmacy technician contacts the facility to obtain the information. As a result, all patients enrolled in the ECP during the study period received this intervention (unless they were rehospitalized or left the SNF before the process was completed), and $54 \%$ of ECP patients required some form of intervention after medication reconciliation was completed (data not shown).

This type of program requires hospital leadership and SNF administrators to be fully committed to developing strong working relationships, and in fact, there is evidence that SNF baseline readmission rates have a greater influence on patients' risk of rehospitalization than the discharging hospital itself. ${ }^{19-21}$ Monthly educational in-services are delivered at the partner SNFs to enhance SNF nursing staff knowledge and clinical acumen. High-impact topics identified by the ECP team include the following: fall prevention, hand hygiene, venous thromboembolism, cardiovascular health, how to report change in condition, and advanced care planning, among others. While no formal pre-post assessments of the SNF nurses' knowledge were conducted, a log of in-services was kept, subjective feedback was collected for performance improvement purposes, and continuing educational units were provided to the SNF nurses who attended.

This study has limitations. As a single-hospital study, generalizability may be limited. While adherence to the program com- 
ponents was closely monitored daily, service gaps may have occurred that were not captured. The program design makes it difficult to quantify the relative impact of the 3 program components on the outcome. Furthermore, the study was observational, so the differences in readmission rates may have been due to unmeasured variables. The decision to enroll patients in the ECP was made by each patient's SNF attending physician, and those who chose to (or not to) participate in the program may manifest other, unmeasured practice patterns that made readmissions more or less likely. Participating physicians also had the option to enroll their patients on a case-by-case basis, introducing further potential bias in patient selection; however, $<5 \%$ of physicians exercised this option. Patients may have also been readmitted to hospitals other than CSMC, producing an observed readmission rate for 1 or both groups that underrepresents the true outcome. On this point, while we did not systematically track these other-hospital readmissions for both groups, there is no reason to believe that this occurred preferentially for ECP or non-ECP patients.

Multiple sensitivity analyses were performed to address the observed differences between ECP and non-ECP patients. These included stratified examinations of variables differing be-

\section{References}

1. Centers for Medicare \& Medicaid Services (CMS), HHS. Medicare Program; Prospective Payment System and Consolidated Billing for Skilled Nursing Facilities (SNFs) for FY 2016, SNF Value-Based Purchasing Program, SNF Quality Reporting Program, and Staffing Data Collection. Final Rule. Fed Regist. 2015;80(149):46389-46477

2. "Readmissions Reduction Program," Centers for Medicare \& Medicaid Services. http://www.cms.gov/Medicare/Medicare-Fee-for-Service-Payment/ AcutelnpatientPPS/Readmissions-Reduction-Program.html. Accessed November 5, 2015.

3. Naylor MD, Brooten D, Campbell R, et al. Comprehensive discharge planning and home follow-up of hospitalized elders: a randomized clinical trial. JAMA. 1999:281:613-620.

4. Naylor MD, Brooten DA, Campbell RL, Maislin G, McCauley KM, Schwartz JS. Transitional care of older adults hospitalized with heart failure: a randomized, controlled trial. J Am Geriatr Soc. 2004;52:675-684.

5. Coleman EA, Parry C, Chalmers S, Min SJ. The care transitions intervention: results of a randomized controlled trial. Arch Intern Med. 2006;166:1822-1828.

6. CMS Office of Information Products and Data Analytics. National Medicare Readmission Findings: Recent Data and Trends. 2012. http://www.academyhealth.org/files/2012/sunday/brennan.pdf. Accessed September 21, 2015.

7. Centers for Medicare \& Medicaid Services, CMS Innovation Center. Initiative to Reduce Avoidable Hospitalizations among Nursing Facility Residents. https://innovation.cms.gov/initiatives/rahnfr/. Accessed November 5, 2015.

8. Unroe KT, Nazir A, Holtz LR, et al. The Optimizing Patient Transfers, Impacting Medical Quality and Improving Symptoms: Transforming Institutional Care Approach: Preliminary data from the implementation of a Centers for Medicare and Medicaid Services nursing facility demonstration project. J Am Geriatr Soc. 2015;65:165-169

9. Ingber MJ, Feng Z, Khatstsky G, et al. Evaluation of the Initiative to Reduce Avoidable Hospitalizations among Nursing Facility Residents: Final Annual Report Project Year 3. Waltham, MA: RTI International, RTI Project Number 0212790.006, January 2016.

10. Ouslander JG, Lamb G, Tappen R, et al. Interventions to reduce hospitalizations from nursing homes: Evaluation of the INTERACT II collaborative quality improvement project. J Am Geriatr Soc. 2011:59:745-753.

11. Kim L, Kou L, Hu B, Gorodeski EZ, Rothberg M. Impact of a Connected Care Model on 30-Day Readmission Rates from Skilled Nursing Facilities. J Hosp Med. 2017;12:238-244.

12. Kansagara D, Englander $H$, Salanitro A, et al. Risk Prediction Models for Hospital Readmission: A Systematic Review. JAMA. 2011:306(15):1688-1698.

13. Averill RF, Goldfield N, Hughes JS, et al. All Patient Refined Diagnosis Relat- tween populations, examination of clustering effects between SNFs, and an analysis adjusted for the propensity to be included in the ECP. The calculated effect of the intervention on readmission remained robust, although we acknowledge that differences in the populations may persist and have influenced the outcomes even after controlling for multiple variables. ${ }^{22-25}$

In conclusion, the results of this intervention are compelling and add to the growing body of literature suggesting that a comprehensive, multipronged effort to enhance clinical oversight and coordination of care for SNF patients can improve outcomes. Given CMS's plans to report SNF readmission rates in 2017 followed by the application of financial incentives in 2018, a favorable climate currently exists for greater coordination between hospitals and SNFs. ${ }^{26}$ We are currently undertaking an economic evaluation of the program.

\section{Acknowledgments}

The authors would like to thank the following people for their contributions: Mae Saunders, Rita Shane, Dr. Jon Kea, Miranda Li, the ECP NPs, the ECP pharmacy team, CSMC's performance improvement team, and Alan Matus.

Disclosure: No conflicts of interest or disclosures.

ed Groups (APR-DRGs): Methodology Overview. 3M Health Information Systems Document GRP-041 (2003). https://www.hcup-us.ahrq.gov/db/nation/ nis/APR-DRGsV20MethodologyOverviewandBibliography.pdf. Accessed November 5, 2015.

14. StataCorp. 2013. Stata Statistical Software: Release 13. College Station, TX: StataCorp LP.

15. Cebul RD, Rebitzer JB, Taylor LJ, Votruba ME. Organizational fragmentation and care quality in the U.S. healthcare system. J Econ Perspect. 2008;22(4): 93-113.

16. Tjia J, Bonner A, Briesacher BA, McGee S, Terrill E, Miller K. Medication discrepancies upon hospital to skilled nursing facility transitions. J Gen Intern Med. 2009;24:630-635.

17. Desai R, Williams CE, Greene SB, Pierson S, Hansen RA. Medication errors during patient transitions into nursing homes: characteristics and association with patient harm. Am J Geriatr Pharmacother. 2011;9:413-422.

18. Chhabra PT, Rattinger GB, Dutcher SK, Hare ME, Parsons KL, Zuckerman IH. Medication reconciliation during the transition to and from long-term care settings: a systematic review. Res Social Adm Pharm. 2012;8(1):60-75.

19. Rahman M, Foster AD, Grabowski DC, Zinn JS, Mor V. Effect of hospital-SNF referral linkages on rehospitalization. Health Serv Res. 2013;48(6, pt 1): 1898-1919.

20. Schoenfeld AJ, Zhang X, Grabowski DC, Mor V, Weissman JS, Rahman M. Hospital-skilled nursing facility referral linkage reduces readmission rates among Medicare patients receiving major surgery. Surgery. 2016;159(5): 1461-1468.

21. Rahman M, McHugh J, Gozalo P, Ackerly DC, Mor V. The Contribution of Skilled Nursing Facilities to Hospitals' Readmission Rate. HSR: Health Services Research. 2017;52(2):656-675

22. Jencks SF, Williams MV, Coleman EA. Rehospitalizations among patients in the Medicare fee-for-service program. New Engl J Med. 2009;360(14): 1418-1428.

23. Hasan O, Meltzer DO, Shaykevich SA, et al. Hospital readmission in general medicine patients: a prediction model. J Hosp Med. 2010;25(3)211-219.

24. Allaudeen N, Vidyarhi A, Masella J, Auerbach A. Redefining readmission risk factors for general medicine patients. J Hosp Med. 2011;6(2):54-60.

25. Van Walraven C, Wong J, Forster AJ. LACE+ index: extension of a validated index to predict early death or urgent readmission after discharge using administrative data. Open Med. 2012;6(3):e80-e90.

26. Protecting Access to Medicare Act of 2014, Pub. L. No. 113-93, 128 Stat. 1040 (April 1, 2014). https://www.congress.gov/113/plaws/publ93/PLAW113publ93.pdf. Accessed October 3, 2015 